\title{
On the definition of energy in general electromagnetic media
}

\author{
A. Nicolet $\dagger$, F. Zolla $\ddagger$ \\ Institut Fresnel, UMR 6133, \\ †Université Paul Cézanne (Aix-Marseille III) \\ †Université de Provence (Aix-Marseille I) Faculté de Saint Jérôme \\ case 162, 13397 Marseille Cedex 20, France \\ Tel.: +33491288 773 - Fax: +33491674 428 \\ e-mail: andre.nicolet@fresnel.fr
}

\begin{abstract}
The definition of the electromagnetic energy density is straightforward in free space but is a much more elusive concept in material media that can be non-linear, anisotropic, dispersive, hysteretic, etc. This paper attempts to give a general approach to the definition of electromagnetic energy density and applies it to various common situations.
\end{abstract}

\section{Introduction}

The definition of the electromagnetic energy plays an important role in electromagnetic modelling. Not only is the knowledge of this energy an important information by itself but it is also useful to set up finite element formulation or to compute auxiliary quantities such as the electromagnetic forces.

The purpose of this paper is to introduce a method as general as possible to define the energy density in a given material. This call 
for the use of differential geometry as it allows a clear and general presentation of both electromagnetic and thermodynamic aspects.

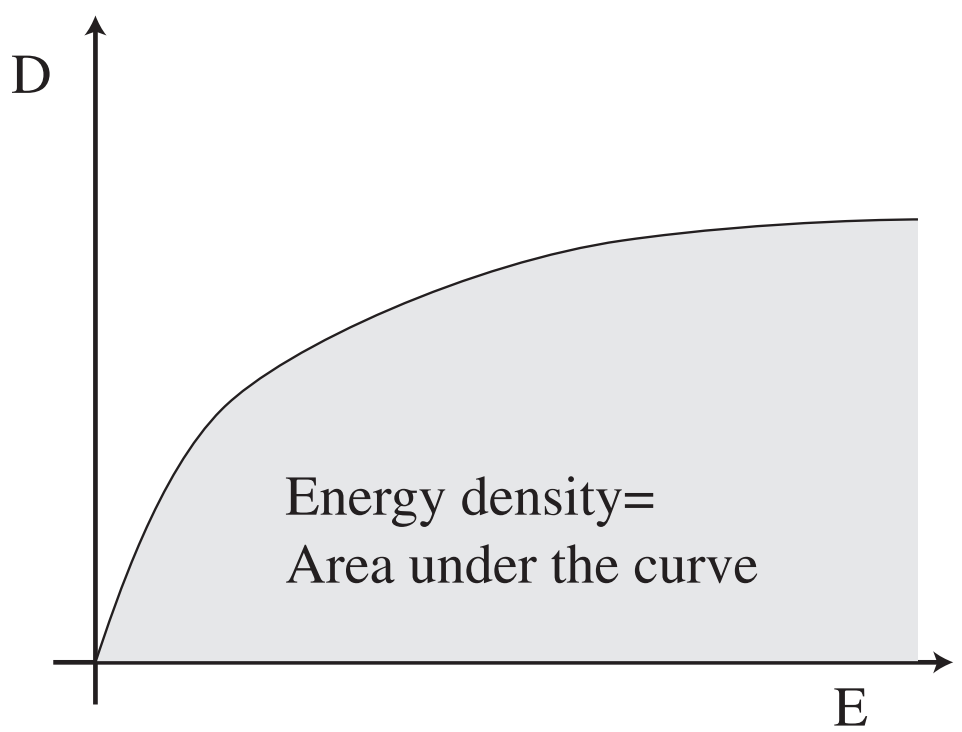

Figure 1: A common presentation is to describe the energy density as the area under a curve "field intensity vs. flux density" but this cannot be a general definition.

\section{Electromagnetic energy}

Using the formalism of differential geometry, Maxwell equations have the following form:

$$
\left\{\begin{aligned}
d H & =J+\partial_{t} D \\
d E & =-\partial_{t} B \\
d D & =\rho \\
d B & =0
\end{aligned}\right.
$$

where $d$ is the exterior derivative (see appendix), the 1-forms $E, H$ are the electric and magnetic fields respectively, the 2-forms $D, B$, and $J$ are the electric flux density or displacement, the magnetic flux density 
or induction, and the electrical current density respectively, and the 3 -form $\rho$ is the electric charge density.

The starting point of energy definition in classical electromagnetism is usually the Poynting identity that can be written, still using differential forms, as:

$$
d(E \wedge H)=J \wedge E+E \wedge \frac{\partial D}{\partial t}+H \wedge \frac{\partial B}{\partial t},
$$

where $\wedge$ is the exterior product (see appendix).

It is quite remarkable that this relation, just as Maxwell equations, does not involve explicitly any metric concept and is completely independent of any material relation. At first sight, it seems to be quite surprising that the law driving the energetic behaviour of the electromagnetic fields does not depend on the properties of the media but, of course, this is hidden in the mixing of $E, H$ with $D, B$ in the same expression.

The various terms in this expression are 3-forms, i.e. power densities. The Poynting 2-form $S \equiv E \wedge H$ is the power flux density (三 means that this equality defines $S$ ) and $P \equiv J \wedge E$ is the exchanged power density, e.g. exchanges with thermal or mechanical process.

As for the last terms, it is convenient to write them as the time derivative of the electromagnetic energy density $W$ :

$$
\frac{\partial W}{\partial t} \equiv E \wedge \frac{\partial D}{\partial t}+H \wedge \frac{\partial B}{\partial t}
$$

It is very easy to write $W$ in free space where the constitutive relations are $D=\varepsilon_{0} * E$ and $B=\mu_{0} * H$ where $*$ is the Hodge star operator involving the metric, and $\varepsilon_{0}$ and $\mu_{0}$ are the dielectric permittivity and magnetic permeability of free space. In this case

$$
W=\frac{1}{2} \varepsilon_{0} E \wedge * E+\frac{1}{2} \mu_{0} H \wedge * H .
$$

The fundamental question is: how to build a general expression for $W$ in the case of a general material?

\section{Constitutive relations}

All the metric aspects of the electromagnetic theory are included in the constitutive laws describing the media behaviour including energy 
storage and dissipation.

A very general form for the constitutive relations can be, for the electric relation (Weiglhofer, 2003):

$$
\mathbf{Y}_{e}[E(\mathbf{x}, t), D(\mathbf{x}, t), H(\mathbf{x}, t), B(\mathbf{x}, t)]=0
$$

and a similar one for the magnetic relation:

$$
\mathbf{Y}_{m}[E(\mathbf{x}, t), D(\mathbf{x}, t), H(\mathbf{x}, t), B(\mathbf{x}, t)]=0
$$

This is a functional relation: it means that the electric field $E$ at a point $\mathbf{x}_{o}$ and time $t_{o}$ may depend on the whole fields $D, H$, and $B$ at any point $\mathbf{x}$ and any time $t \leq t_{o}$. Physical quantities are vector fields:

$$
E, D, H, B \in \mathbb{H}=\left\{(\mathbf{x}, t) \in \mathbb{R}^{3} \times \mathbb{R} \rightarrow \mathbb{R}^{3}\right\}
$$

that are elements of some functional space $\mathbb{H}$ (e.g. square integrable vector fields with square integrable convenient combinations of partial derivatives...). General constitutive laws:

$$
\begin{aligned}
D(\mathbf{x}, t) & =\mathcal{D}(E, H, \mathbf{x}, t) \\
B(\mathbf{x}, t) & =\mathcal{B}(E, H, \mathbf{x}, t)
\end{aligned}
$$

are functionals of the vector fields $E$ and $H$ :

$$
\mathcal{D}, \mathcal{B} \in\left\{\mathbb{H}^{2} \times \mathbb{R}^{3} \times \mathbb{R} \rightarrow \mathbb{R}^{3} \times \mathbb{R}\right\}
$$

and it is only a very particular case that, for instance, the electric displacement at position $\mathbf{x}$ and time $t$ only depends a priori on the electric field value at these very space-time co-ordinates.

The only global general constraint is causality: only the points of space-time in the past light cone can have an influence. Of course, usual material laws are often more simple than the most general case but may anyway remain quite complicated:

- A material is non-local in space if the property of this material on a point depends on other points of space (or on spatial derivatives).

- A material is non-local in time, non-instantaneous or dispersive if the property of this material at a particular time depends on other instants (or on time derivatives). 
- A material is non-homogeneous if the formal relation $\mathbf{Y}$ depends on the point of space which is considered.

- A material is non-stationary or non-homogeneous in time if the formal relation $\mathbf{Y}$ depends on the instant which is considered.

- A material is non-linear if the response to the sum of any set of excitations is not the sum of the responses to each excitation.

- A material is anisotropic if the relation can not be reduced to a scalar relation and bianisotropic if it mixes electric and magnetic quantities.

Free space is a local instantaneous homogeneous stationary linear isotropic medium. The properties may in fact depend on the particular homogenisation process: the space cell used to smooth the field is usually large enough to absorb all remote effects and it eliminates the non-local character in space. On the contrary, it is common to encounter dispersive, non-linear, anisotropic media and also hysteretic media with a long time memory (so important for modern data storage).

\section{Thermodynamic structure of elec- tromagnetism}

Thermodynamic is usually introduced for discrete systems composed of boxes with a finite volume. The first principle expressing the energy conservation is written $d U=T d S-P d V$ where $U$ the internal energy, $S$ the entropy, and $V$ the volume are extensive variables and $T$ the temperature and $P$ the pressure are intensive variables. All these variables may be considered as co-ordinates in a thermodynamic space (or "energetic" space) and $d$ is nothing else but the exterior derivative in this space where derivation is taken with respect to these thermodynamic variables. Note that the expression for $d U$ implies that the extensive variable $U$ is a function $U(S, V)$ of the extensive variables $S$ and $V$. It is therefore homogeneous of degree 1 in the sense of Euler and we have $U=T S-P V$. Just as in the case of the Poynting identity, we have a general relation for energy independent of any 
particular substance but here again the information is hidden in the mixing of $S, V$ together with $P, T$ in the same expression and a state equation for the particular substance is necessary to go further.

Given a thermodynamic cycle $\gamma$, e.g. in a thermal machine, $\int_{\gamma} d U=$ 0 so that $\int_{\gamma} T d S=\int_{\gamma} P d V$ where the lefthand side is the thermal energy brought to the system and the righthand term the recuperated mechanical work.

More generally, pairs of conjugate variables, an extensive one together with a corresponding intensive one, are introduced to describe the various energetic aspects of a system.

In a continuous media, extensive variables turn to densities (i.e. 3 -forms with respect to the space variables $x, y, z)$ such as the energy density $u$, the entropy density $s \ldots$ and intensive variables turn to scalar fields (i.e. 0 -forms with respect to the space variables $x, y, z$ ) such as the temperature field $T$... To generalize to vector quantities, it must be noticed that the exterior product of a 1 -form by a 2 -form is just a duality product where corresponding components are multiplied together and added (see appendix). The result is a density (3-form) that will contribute to the internal energy density $u$. As a practical rule, it can be observed that conjugate thermodynamic variables are usually dual tensor variables so that their duality product involved in the energy definition has a simple algebraic form.

The problem with electromagnetic vector quantities is that a power density is obtained instead of directly an energy density.

A possibly dispersive, anisotropic, non-linear electric relation is considered here but local in space, stationary, and not bianisotropic. A possible definition for the electric part of the energy density would be:

$$
W_{e} \equiv \int_{0}^{t} E \wedge \frac{\partial D}{\partial t^{\prime}} d t^{\prime}=\int_{0}^{t}\left(\sum_{i=1}^{3} E_{i} \frac{\partial D_{i}}{\partial t^{\prime}}\right) d t^{\prime} .
$$

where the $E_{i}, D_{i}$ are the components in any particular co-ordinate system. The time derivative of this expression certainly gives the power density but what we are looking for are the general conditions which guarantee that $W_{e}(D)$ is a state variable, i.e. that it only depends on the current state of the field $D$ and not on the whole process between the reference time 0 and the current time $t$. If it is the case, 
Eq. 3 must at least be a curvilinear integral in the "energetic" space (where the co-ordinates are the thermodynamical variables including the electromagnetic ones). From now, all the differential geometric expressions concern this "energetic" space.

$E$ is a 1 -form and $D$ is a 2 -form in space-time but $E$ is a (vector valued) function of (the components of) $D$ in the "energetic" space describing the local thermodynamic equilibrium of a homogenisation cell of the material. The "energetic" 1-form $E d D\left(=\sum_{i=1}^{3} E_{i} d D_{i}\right.$ in a particular space-time co-ordinate system) is the electric contribution to the internal energy density differential just as $T d s$ is the thermal contribution. This means that in a Cartesian co-ordinate system $x, y, z$, $E d D=E_{x} d D_{x}+E_{y} d D_{y}+E_{z} d D_{z}$ but also that, for instance, in a spherical co-ordinate system $r, \theta, \phi, E d D=E_{r} d D_{r}+E_{\theta} d D_{\theta}+$ $E_{\phi} d D_{\phi}$. Such a form is even valid for non-orthogonal systems of co-ordinates!

A necessary condition for $W_{e}(D)$ to be a state function of the state variable $D$ is that $E d D$ is a closed form: $d(E d D)=d E \wedge d D=0$. In this case, it is licit to write $E d D=d W_{e}$ as an exact form. In a particular space-time co-ordinate system, these conditions become $\frac{\partial E_{i}}{\partial D_{j}}-\frac{\partial E_{j}}{\partial D_{i}}=0$ for $i \neq j$. Therefore, in the case of a linear anisotropic electric relation (necessarily lossless), this implies the symmetry of the permittivity tensor.

In the case of isotropic media where the relation is simply between the moduli of $E$ and $D$, it is asserted that the dissipated energy is the surface of the loops in the " $E-D$ plane". Consider here a twodimensional surface $\Sigma$ in the (at least) 6-dimensional $E-D$ "energetic" space and its boundary $\partial \Sigma$ forming a closed loop. By the Stokes theorem $\int_{\Sigma} d E \wedge d D=\int_{\partial \Sigma} E d D=0$ and the curvilinear integral in the "energetic" space is independent of the path hence gives a state variable. The 2-form $d E \wedge d D$ (plus all the other terms coming from the various contributions to the energy) gives in fact a symplectic structure (Balian, 2001) to the "energetic" space. Some canonical transformations that leave this structure invariant can be performed such as the Legendre transformation which consists in replacing $D$ by $E$ as the variable to obtain the co-energy $W_{e}^{\prime}(E)$.

In a particular material, three situations can be encountered. The 
two first ones correspond to the case where Eq. 3 is a curvilinear integral:

- Eq. 3 is a curvilinear integral and $E d D$ is a closed form, the electric energy is easily defined as a state variable

- Eq. 3 is a curvilinear integral and $E d D$ is not a closed form thus there is some dissipation but this one can be easily computed on a cycle as a flux integral of $d E \wedge d D$.

- The third case is even worse since Eq. 3 is not even a curvilinear integral: it depends not only on the path in the "energetic" space but also on the choice of the parameters, e.g. the time taken to run from the initial state to the final state. This third situation arise, for instance, in the cases where $D$ depends on $\frac{\partial E}{\partial t}$ or on delayed values $E\left(t-t_{i}\right)$.

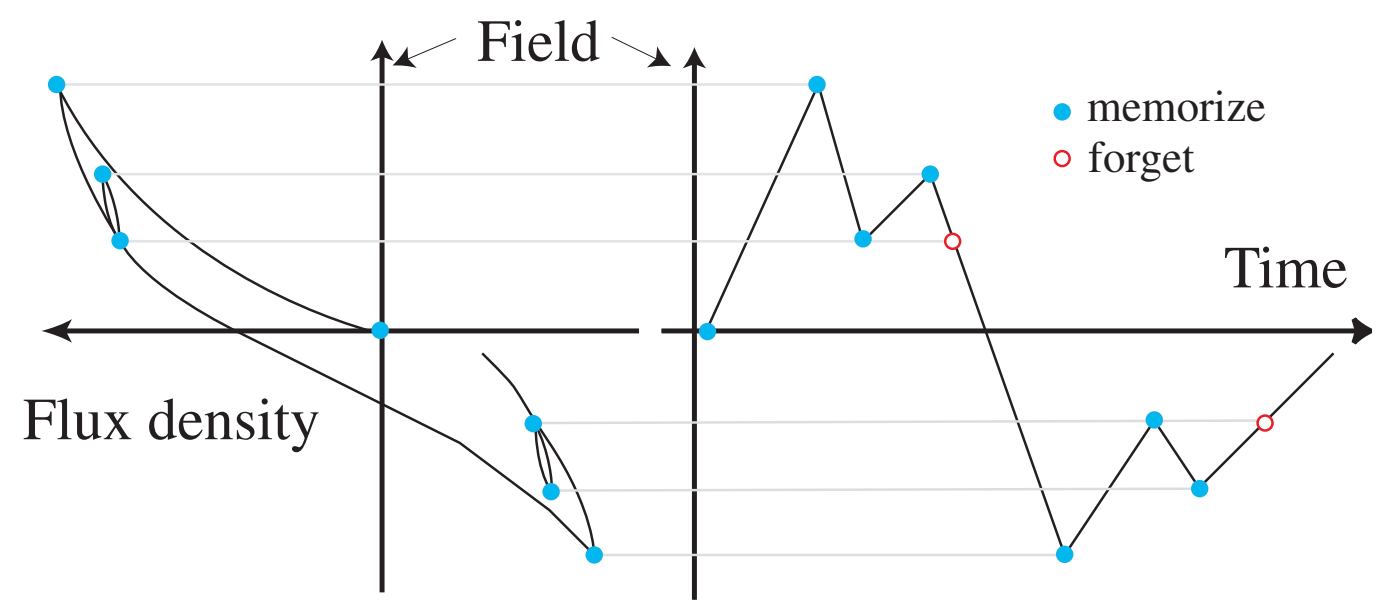

Figure 2: In a Preisach-like model, the constitutive law depend on a discrete set of historical dates determined by a particular memorization-erasure process specific to the material.

Linear non-dispersive and non-dissipative materials are an example of the first case. 
An interesting example of the second case is the Preisach model (Mayergoyz, 1991) introduced to model ferromagnetic hysteresis. In this case, the relation depends on a history, i.e. fixed dates in the past (as opposed to delayed values running with the time). In this case, the integral in Eq. 3 is a curvilinear integral between the fixed dates that form a null measure set and the energy dissipated on cycles can be estimated. If the instantaneous dissipation has to be estimated, some supplementary hypotheses compatible with the cycle dissipation are necessary (Delincé, 2004).

As a example of the third situation, the case of a simple dispersive and dissipative dielectric material is given. Such materials are often modelled by (Jackson, 1999) :

$$
\left\{\begin{array}{l}
D=\varepsilon_{0} E+P \\
\alpha P+\beta \frac{\partial P}{\partial t}+\gamma \frac{\partial^{2} P}{\partial t^{2}}=E
\end{array}\right.
$$

where the second equation describes the polarization process, i.e. the way microscopic dipoles react to the varying electric field $E$. $\alpha, \beta$, and $\gamma$ are constants depending on the considered material. The coefficient $\alpha$ describes the elastic behaviour, $\beta$ the dissipation, and $\gamma$ the inertia. These coefficients are constants depending on the considered material. The power density is given by:

$$
\begin{gathered}
E \cdot \frac{\partial D}{\partial t}=E \cdot \varepsilon_{0} \frac{\partial E}{\partial t}+\left(\alpha P+\beta \frac{\partial P}{\partial t}+\gamma \frac{\partial^{2} P}{\partial t^{2}}\right) \cdot \frac{\partial P}{\partial t}= \\
\frac{1}{2} \frac{\partial\left(\varepsilon_{0} E^{2}+\alpha P^{2}+\gamma\left(\frac{\partial P}{\partial t}\right)^{2}\right)}{\partial t}+\beta\left|\frac{\partial P}{\partial t}\right|^{2}
\end{gathered}
$$

Obviously, the energy density $W_{e}=\frac{1}{2}\left(\varepsilon_{0} E^{2}+\alpha P^{2}+\gamma\left(\frac{\partial P}{\partial t}\right)^{2}\right)+$ $\beta \int_{0}^{t}\left|\frac{\partial P}{\partial \tau}\right|^{2} d \tau$ depends on the time variation of the process and cannot be incorporated as a simple state function in the thermodynamic description of the system.

\section{Conclusion}

A general thermodynamic framework to define the electromagnetic energy density as a state variable is given. The use of differential 
geometry is quite natural since there is no natural metric in the "energetic" space but rather a symplectic structure and it is useful since in the anisotropic case the working space is at least 6-dimensional.

It must be noticed that most hysteresis models give the field intensity - flux density behaviour but do not separate explicitly stored and dissipated energy as they should do but rely on the "surface of closed loops" recipe.

The concept of energy density is not so obvious in sophisticated materials and deserves at least a careful definition.

\section{Appendix: differential geometry}

Given a $n$-dimensional space with a (global) co-ordinate system $u_{1}, \cdots, u_{n}$ (not necessarily orthogonal), the exterior derivative $d$ of a function $f\left(u_{1}, \cdots u_{n}\right)$ is its differential $d f=\sum_{i} \frac{\partial f}{\partial u_{i}} d u_{i}$. This is a 1-form. A general 1-form can be written $\sum_{i} g_{i}\left(u_{j}\right) d u_{i}$ where $g_{i}\left(u_{j}\right)$ are functions of the co-ordinates $u_{j}$. If a 1-form can be expressed as the differential of a function, it is an exact 1-form.

A curve $\gamma$ is an application from an interval $I=\left[t_{0}, t_{1}\right]$ of $\mathbb{R}$ on the $n$-dimensional space: $\mathbf{r}(t)=\left(u_{1}(t), \cdots, u_{n}(t)\right)$ where $t$ is the parameter. The integral $\int_{\gamma} \alpha$ of a 1 -form $\alpha=\sum_{i} g_{i}\left(u_{j}\right) d u_{i}$ on the curve $\gamma$ is defined by $\int_{\gamma} \alpha=\int_{t_{0}}^{t_{1}}\left(\sum_{i} g_{i}\left(u_{j}(t)\right) \frac{d u_{i}(t)}{d t}\right) d t$. The value of the integral does not depend on the choice of the parameter and the integral of an exact form $d f$ depends only on the boundary points: $\int_{\gamma} d f=f\left(\mathbf{r}\left(t_{1}\right)\right)-f\left(\mathbf{r}\left(t_{0}\right)\right)$. Therefore, the integral of an exact form on a closed curve (for which $\mathbf{r}\left(t_{1}\right)=\mathbf{r}\left(t_{0}\right)$ ) is equal to zero.

The exterior product $\wedge$ is the skew-symmetric tensor product such that $d u_{i} \wedge d u_{j}=-d u_{j} \wedge d u_{i}=\frac{1}{2}\left(d u_{i} \otimes d u_{j}-d u_{j} \otimes d u_{i}\right)$. A general 2form is a linear combination $\sum_{i, j} g_{i j} d u_{i} \wedge d u_{j}$. The exterior derivative of the 1 -form $\sum_{i} g_{i} d u_{i}$ is $d \sum_{i} g_{i} d u_{i}=\sum_{i, j} \frac{\partial g_{j}}{\partial u_{i}} d u_{i} \wedge d u_{j}$. A 2 -form $\beta$ is exact if it can be expressed as the exterior derivative of a 1-form.

A surface $\Sigma$ is an application from a two-dimensional open domain $\Omega \subset \mathbb{R}^{2}$ on the $n$-dimensional space: $\mathbf{r}(s, t)=\left(u_{1}(s, t), \cdots, u_{n}(s, t)\right)$ where $s, t$ are the parameters. The integral $\int_{\Sigma} \beta$ of a 2 -form $\beta=$ 
$\sum_{i, j} g_{i j} d u_{i} \wedge d u_{j}$ on the surface $\Sigma$ is defined by

$$
\int_{\Sigma} \beta=\iint_{\Omega} \sum_{i, j}\left(g_{i j} \frac{\partial\left(u_{i}, u_{j}\right)}{\partial(s, t)}\right) d s d t
$$

where $\frac{\partial\left(u_{i}, u_{j}\right)}{\partial(s, t)}$ are the Jacobians. The value of the surface (flux) integral depends on $\Sigma$ but not on the way the parameters are chosen.

The Stokes theorem states that $\int_{\Sigma} d \alpha=\int_{\partial \Sigma} \alpha$ where $\partial \Sigma$ is the boundary (curve) of the surface $\Sigma$.

More generally $p$-forms (with $0 \leq p \leq n$ ) are defined as totally skew-symmetric tensors and can be manipulated using exterior derivative and product. Given a 1-form $\alpha=\alpha_{1} d u_{1}+\alpha_{2} d u_{2}+\alpha_{3} d u_{3}$ and a 2 -form $\beta=\beta_{23} d u_{2} \wedge d u_{3}+\beta_{31} d u_{3} \wedge d u_{1}+\beta_{12} d u_{1} \wedge d u_{2}$, one has for instance: $d \beta=\left(\frac{\partial \beta_{23}}{\partial u_{1}}+\frac{\partial \beta_{31}}{\partial u_{2}}+\frac{\partial \beta_{12}}{\partial u_{3}}\right) d u_{1} \wedge d u_{2} \wedge d u_{3}$ and $\alpha \wedge \beta=\left(\alpha_{1} \beta_{23}+\alpha_{2} \beta_{31}+\alpha_{3} \beta_{12}\right) d u_{1} \wedge d u_{2} \wedge d u_{3}$.

All the concepts here above rely only on the differential structure of the space. Supplementary structures are often introduced in the form of given special tensors.

The symplectic structure on an even-dimensional space is determined by a 2 -form $\omega$ (i.e. a rank 2 covariant skew-symmetric tensor) that is closed $(d \omega=0)$ and non-degenerate. There is always (at least) a co-ordinate system such that $\omega=d u_{1} \wedge d u_{2}+\cdots+d u_{2 n-1} \wedge d u_{2 n}$. This structure is characteristic of phase spaces where canonical transformations are the ones preserving the symplectic structure.

The metric structure is determined by a rank 2 covariant symmetric tensor $\mathbf{g}$ whose $n^{2}$ coefficients form a positive definite matrix. Contrarily to symplectic structure, there is freedom in the choice of this metric. The metric allows the definition of a Hodge star operator * that is a linear operator on differential forms mapping $p$-forms on $(n-p)$-forms.

Particular cases of spaces with a metric are the Euclidean spaces $\mathbb{E}^{n}$ where Cartesian co-ordinates can be chosen so that the coefficients of the metric form a unit matrix. For $\mathbb{E}^{3}$, Cartesian co-ordinates are denoted $\left\{u_{1}=x, u_{2}=y, u_{3}=z\right\}$ and the metric has the form: $\mathrm{g}=d x \otimes d x+d y \otimes d y+d z \otimes d z$. In these Cartesian co-ordinates, the Hodge operator has the following action:

$* d x=d y \wedge d z, * d y=d z \wedge d x, * d z=d x \wedge d y$ 
$*(d x \wedge d y)=d z, *(d z \wedge d x)=d y, *(d y \wedge d z)=d x$

$* 1=d x \wedge d y \wedge d z, *(d x \wedge d y \wedge d z)=1$.

\section{References}

Weiglhofer, W., Lakhtakia, A. (2003), Introduction to Complex Mediums for Optics and Electromagnetics", SPIE Press (Bellingham, Washington USA).

Balian, R., Valentin, P. (2001), "Hamiltonian structure of thermodynamics with gauge", Eur. Phys. J. B 21, 269-282 [cond-mat/0007292]. Delincé, F. (1994), "Modélisation des régimes transitoires dans les systèmes comportant des matériaux magnétiques non linéaires et hystérétiques", Ph. D. Thesis, University of Liège, 1994.

Delincé, F., Nicolet, A., Genon, A., Legros, W. (1994), "Analysis of ferroresonance with a finite element method taking hysteresis into account", Journal of Magnetism and Magnetic Materials, 133, 557560.

Mayergoyz, I. (1991), Mathematical Models of Hysteresis, SpringerVerlag, New York.

Jackson, J.D. (1999), Classical Electrodynamics, 3rd ed., Wiley, NewYork. 